\title{
AIDS tendencies in São Mateus microregion, State of Espírito Santo, and Brazil from 1999 to 2008
}

\author{
Ana Paula Martins Vilela ${ }^{[1]}$, Franciéle Marabotti Costa Leite ${ }^{[1]}$, Edilson Romais Schmildt ${ }^{[1]}$, \\ Simone Mendes Carvalho ${ }^{[1]}$, Susana Bubach ${ }^{[1]}$, Kamila Medani Tristão ${ }^{[1]}$ \\ and Emanuelle Pires de Amorim ${ }^{[1]}$
}

[1]. Curso de Enfermagem, Centro Universitário Norte do Espírito Santo, Universidade Federal do Espírito Santo, São Mateus, ES.

\begin{abstract}
Introduction: In Brazil, AIDS has been described as a multi-faceted pandemic. This study aimed to describe the trends of AIDS in São Mateus microregion, in Espírito Santo, and in Brazil, from 1999 to 2008. Methods: Data were collected from the Notification Offences System, and a trend analysis was made. Results: Microregion-based results are close to the state and national levels but with particular features that indicate the presence of regional sub-epidemics. Conclusions: Despite progress in nearly thirty years of the epidemic, AIDS remains an incurable disease, and prevention is still the best defense against it together with the implementation of specific public policies.
\end{abstract}

Keywords: Public health. Epidemiology. Acquired immunodeficiency syndrome.

The identification of acquired immune deficiency syndrome (AIDS) in 1981 became a milestone of mankind history ${ }^{1}$. In 1985, AIDS was recognized by the Health Ministry (HM) as an emergent problem of public health, becoming an issue requiring public notification ${ }^{2}$. At present, it is undoubtedly one of the most serious health problems presenting particular characteristics, which demands continuous study of all its occurrence and propagation aspects ${ }^{3}$.

Its reach does not respect borders, sex, ethnicity, or social condition ${ }^{4}$, thus being considered a phenomenon. Its occurrence is known in different parts of the world as regional epidemics with decisive and particular characteristics ${ }^{5}$.

The epidemic goes through different stages that could determine specific policies to prevent and control the disease, as well as the ways people could be made aware of in dealing with the disease and especially with infected people ${ }^{4}$. Initially, it occurred most evidently in specific groups, such as men having sex with men (MSM), the youth of upper social classes, prostitutes, and intravenous drug users ${ }^{6}$. However, the profile has been changing, and it is undergoing a transition, marked by processes such as: feminization, aging, pauperization, heterosexualization, and interiorization of the epidemic ${ }^{3}$.

Therefore, the knowledge on the peculiarity of epidemic's manifestation is justified, in relation to decisive and constraint aspects of population vulnerability, because it is fundamental for consistency and effectiveness for better prevention and to control actions. Thus, this study aimed at describing the AIDS tendencies in São Mateus microregion, State of Espírito Santo,

Address to: Ana Paula Martins Vilela. Avenida México 44, Bairro Quitandinha, 35180-070 Timóteo, MG, Brasil.

Phone: 5531 3849-5381; 5531 9253-4438

e-mail: anapaulavilela@hotmail.com

Received in 17/12/2010

Accepted in 29/09/2011 and Brazil, from 1999 to 2008, through general occurrence rate, occurrence rate by sex, and sex ratio. This study also aimed to compare the regional tendency profiles between the state and the national ones.

A descriptive, retrospective, and ecological study with temporal series and longitudinal limits was conducted in July and August of 2010. The study started with gathering of information from the Notification Offences Information System available in the HM (Health Ministry) site of STD (Sexually Transmitted Disease) National Program and AIDS ${ }^{7}$.

The research population includes the whole São Mateus microregion, which is in the north of Espírito Santo, situated southeast of Brazil with 3,352,024 inhabitants and 78 towns $^{8}$. In 2008, that microregion presented a contingent population of 243,641 inhabitants; it consists of 9 towns: Jaguaré, Boa Esperança, São Mateus, Pedro Canário, Conceição da Barra, Montanha, Pinheiros, and Ponto Belo e Mucuricí. Most of them are small towns ( 8 with less than 50,000 inhabitants and 1 with more than 100,000 habitants) with the worst HDI (Human Development Index) in the state 9

The historical series data from 1999 to 2008 were analyzed referring to AIDS cases diagnosis to São Mateus microregion, State of Espírito Santo, and Brazil, comparing the profile of that area with the state and with the country as a whole. To trace the epidemic profile, many variables were selected: number of diagnosed cases and diagnosed sexes and AIDS occurrence rates. AIDS occurrence rates by sex and sex ratio were calculated in the microregion as well as in the State of Espírito Santo and Brazil.

The AIDS occurrence rates and AIDS occurrence rates by sex of every analyzed year were gathered from the number of diagnosed AIDS cases and the number of AIDS cases diagnosed by sex in every year of historical series divided by the resident population of the studied year gathered from Datasus database ${ }^{7}$. All the occurrence rates were expressed by 100,000 inhabitants. 
The sex ratio calculation was done by dividing the number of diagnosed AIDS cases in the male sex by the number of diagnosed cases in the female sex in every studied year. All those calculations were done using Microsoft Excel, version 7.0.

The tendency analysis was done through polynomial regression model by using the statistical program Genes ${ }^{10}$, considering the following: dependent variables (Y) occurrence rate of diagnosed AIDS cases, occurrence rate by sex of diagnosed AIDS cases, and sex ratio of diagnosed AIDS cases; independent variable $(\mathrm{X})$ - the diagnosis year of those indexes. The polynomial regression test was done to identify the probability value in each degree and to select the tendency line degree, which is adjusted the best. The selection was done until degree 3, and all analyses were done with $1 \%$ level of significance.

Finally, the tendency lines of each variable were built by using Microsoft Excel, according to the degree selected by the test. The occurrence diagnosis years were identified in the graph from 1 to 10: the number 1 referring to the year 1999, 2 to 2000 , and so on. For the data analysis, the determination coefficients $\left(\mathrm{R}^{2}\right)$ with values lower than $0.5(50 \%)$ are considered indicators of low data adjustment to the regression line, showing weak tendency relation. The increase or decrease of tendency intensity was evaluated through a comparison of the generated tendency line equations.

The date used came from public use and access database. Therefore, permission from the Research Ethics Committee was not necessary.
Figure 1 presents the occurrence rates of AIDS cases in São Mateus microregion, State of Espírito Santo, and Brazil from 1999 to 2008. In the microregion $\left(\mathrm{R}^{2}=0.6184\right)$ and in Brazil $\left(R^{2}=0.7308\right)$, increasing tendency of AIDS cases was evident with medium occurrence rate of 11.73 cases out of 100,000 inhabitants and 18.92 cases out of 100,000 inhabitants, respectively. The increasing tendency of occurrence rate in the microregion was larger than in Brazil. Nevertheless, the State of Espírito Santo $\left(R^{2}=0.6287\right)$ presented decreasing tendency of AIDS cases, with medium occurrence rate of 17.42 cases out of 100,000 inhabitants.

As for sex ratio (male/female), an increasing tendency was found in the microregion only but with weak relation tendency $\left(\mathrm{R}^{2}=0.3604\right)$. Differently, the State of Espírito Santo $\left(\mathrm{R}^{2}=0.8136\right)$ and Brazil $\left(\mathrm{R}^{2}=0.9773\right)$ presented decreasing sex ratio tendencies with determination coefficient values that demonstrated high adjustment to the regression line. The decreasing tendency intensity of sex ratio was larger in the State of Espírito Santo than in Brazil. The medium sex ratios were 1.24, 1.3, and 1.55 in the microregion, State of Espírito Santo, and Brazil, respectively (Figure 2).

The three analyzed areas presented increasing tendency of occurrence rate by sex in both sexes. The increasing tendency intensity of occurrence rate in the female sex is by the following decreasing order: microregion, state of Espírito Santo, and Brazil. Nevertheless, the increasing tendency intensity of occurrence rate in the male sex is by the following decreasing order: microregion, Brazil, and State of Espírito Santo.
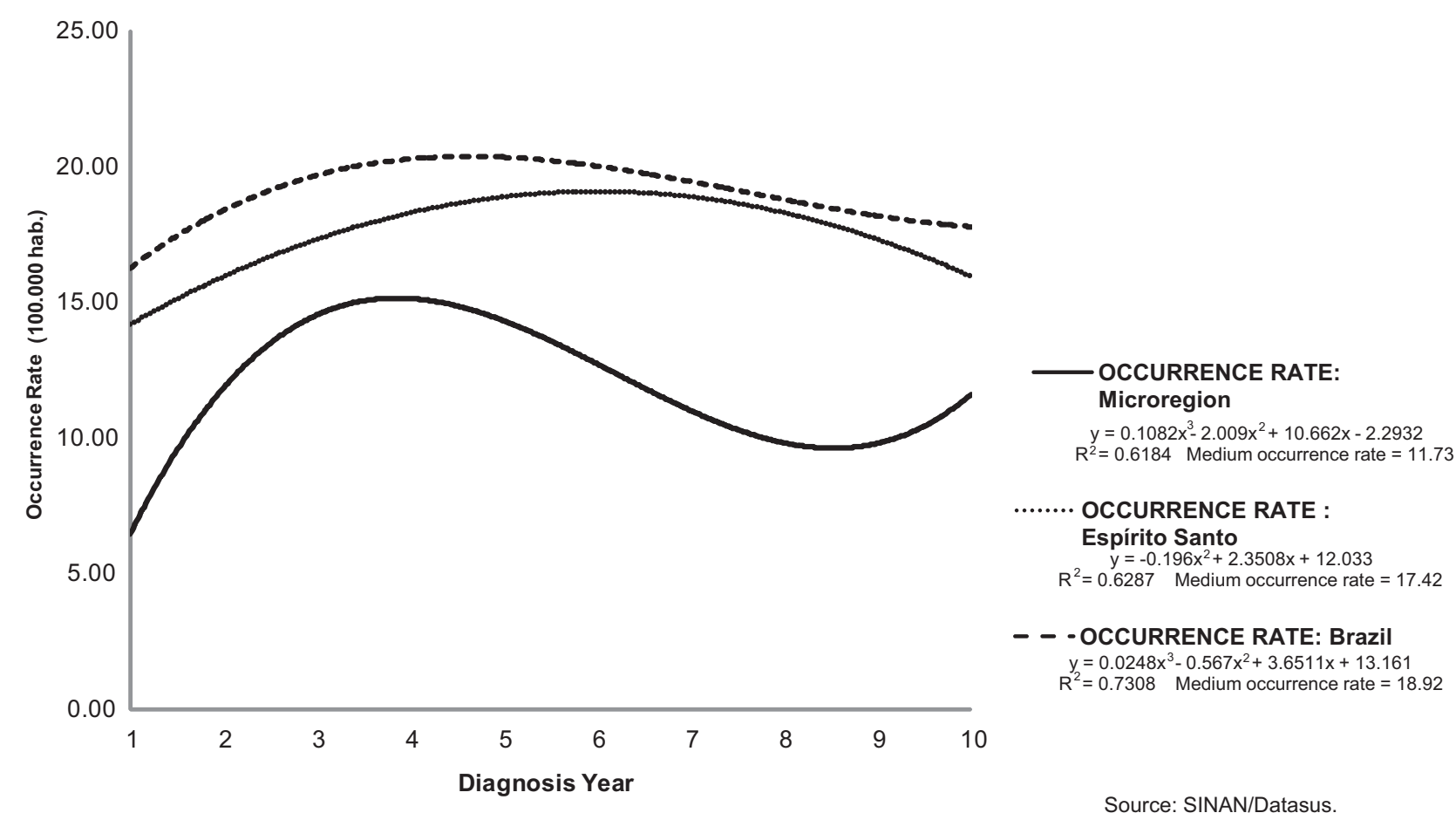

FIGURE 1 - Occurrence rate of diagnosed AIDS cases in São Mateus microregion, State of Espirito Santo, and Brazil, from 1999 to 2008. 


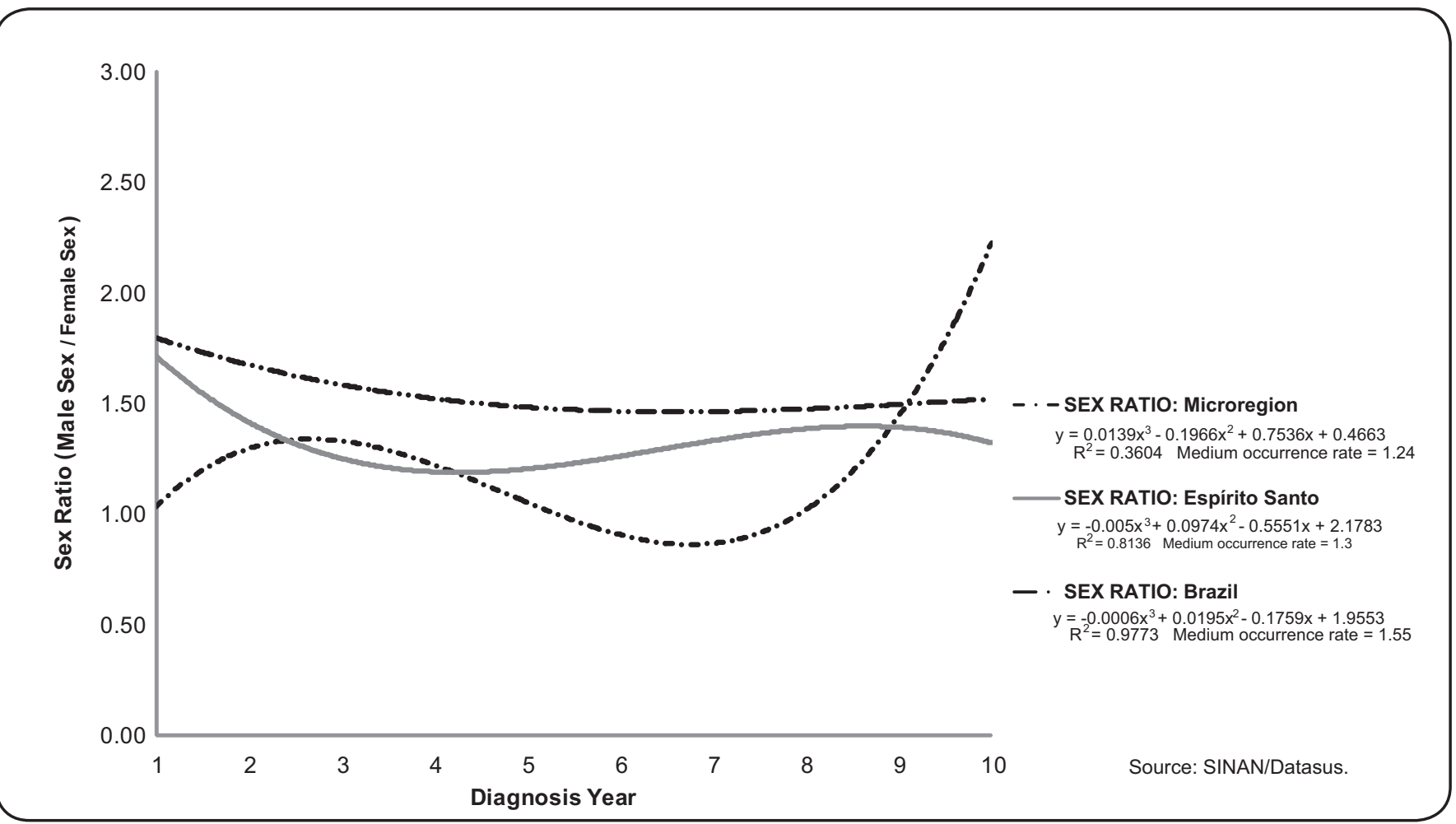

FIGURE 2 - Sex ratio in São Mateus microregion, State of Espírito Santo, and Brazil, from 1999 to 2008.

The medium occurrence rate in the microregion was 11.86 cases out of 100,000 male inhabitants and 11.60 cases out of 100,000 female inhabitants. In the state of Espírito Santo, 20.04 cases out of 100,000 inhabitants were found in male sex and 14.84 cases out of 100,000 inhabitants in female sex. Brazil presented 23.31 cases out of 100,000 inhabitants in male sex and 14.66 cases out of 100,000 inhabitants in female sex (Figure 3).

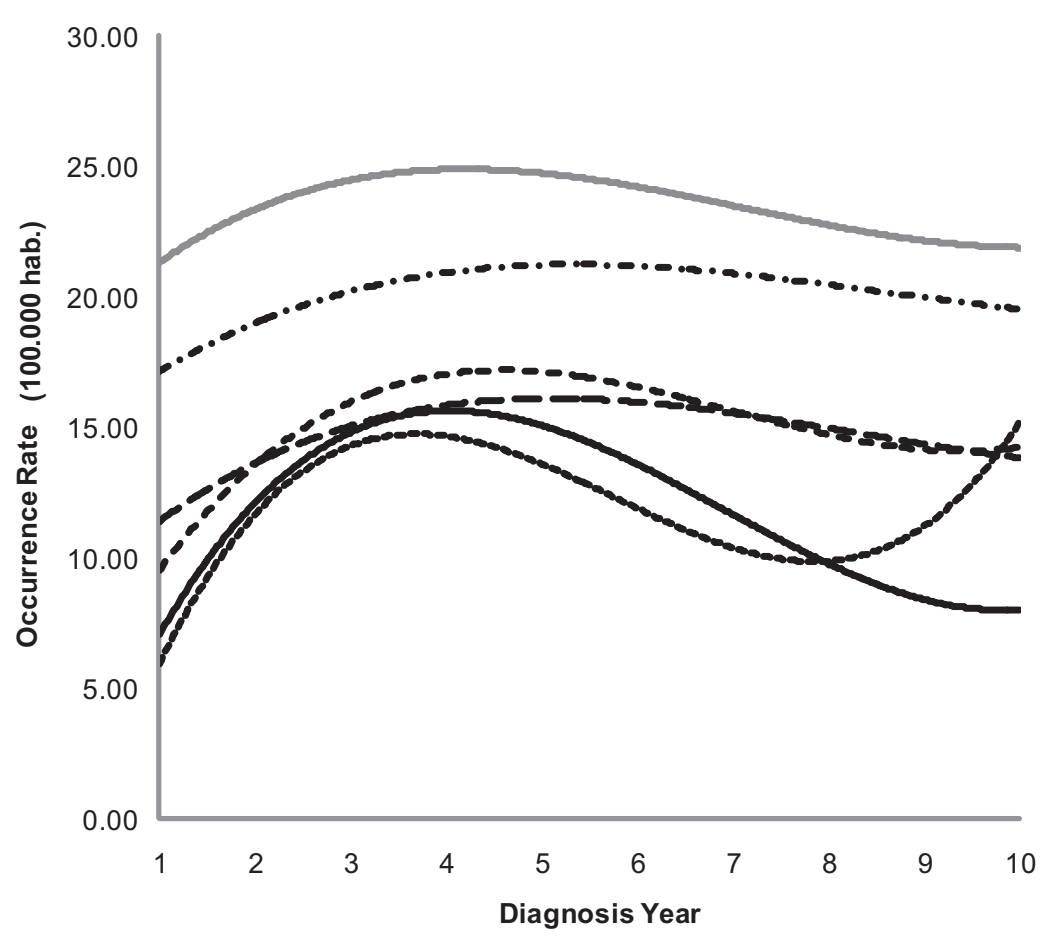

Microregion: Female Sex

$y=0.0798 x^{3}-1.6562 x^{2}+9.4672 x-0.8555$ $R^{2}=0.3034$ Medium occurrence rate $=11.60$

- - - Microregion: Male Sex

$y=0.1366 x^{3}-2.3626 x^{2}+11.86 x-3.7286$ $R^{2}=0.5589$ Medium occurrence rate $=11.86$

- - Espírito Santo: Male Sex

$y=0.0154 x^{3}-0.3966 x^{2}+2.921 x+14.594$ $R^{2}=0.5577$ Medium occurrence rate $=20.04$

- - Espírito Santo: Female Sex $y=0.0555 x^{3}-1.1639 x^{2}+7.1683 x+3.4281$ $\mathrm{R}^{2}=0.7824 \quad$ Medium occurrence rate $=14.84$

\section{- Brazil: Female Sex}

$y=0.0206 x^{3}-0.5116 x^{2}+3.6073 x+8.2687$ $R^{2}=0.8238$ Medium occurrence rate $=14.66$

\section{Brazil: Male Sex}

$y=0.0293 x^{3}-0.6277 x^{2}+3.7148 x+18.176$ $R^{2}=0.6541$ Medium occurrence rate $=23.31$

Diagnosis Year

Source: SINAN/Datasus.

FIGURE 3 - Occurrence rate by sex of AIDS cases in São Mateus microregion, state of Espírito Santo, and Brazil according to the diagnosis year, from 1999 to 2008. 
Brazil has been among the top countries worldwide with the highest number of AIDS cases since the epidemic began. It happened first in Latin America, with more than $40 \%$ $(730,000)$ of the people infected ${ }^{11}$.

Although there is a study ${ }^{12}$ showing that health politics adopted by the Brazilian government resulted in stabilization and even AIDS reduction in Brazil, the occurrence rate of the epidemic still shows that AIDS is not highly prevented in the country; thus, this disease needs greater attention from Brazilian authorities. New-infection rate decreased in several countries, but those favorable tendencies are somewhat globally compensated by new-infection rate increase in other countries, especially in underdeveloped countries or in developing ones, such as Brazil ${ }^{11}$.

The difference in the tendency profile of AIDS occurrence rates between the state of Espírito Santo and the microregion, consisting of nine towns, shows that the continental dimensions of Brazil create important regional differences regarding the epidemic profile in the country ${ }^{13}$. That fact also indicates the recent epidemic interiorization process mentioned by several studies ${ }^{8}$, in which the case occurrence circumscription in the great urban centers gradually spread to a greater geographical area where the infection has reached, reaching areas less urbanized and populous in Brazil, such as São Mateus microregion, consisting of small towns, considered countryside with the worst state HDI. In 2000, indexes were below 0.730 and under the state average $(0.767)^{9}$. Besides, it is early to evaluate the impact of decreasing AIDS occurrence tendency rates in the state of Espírito Santo once it is known that there is a big delay in the notifications and information on the way to the central level of Epidemiological Surveillance System ${ }^{13}$.

Current epidemic indicators show that the epidemic profile of patients living with AIDS/HIV (human immunodeficiency virus) has been changing and going through a transition period, marked by processes such as: feminization, aging, pauperization, and heterosexualization ${ }^{3}$.

Heterosexual relationship is the mode of transmission that contributes the most to the wide feminization in the country ${ }^{14}$. Although until the present days there are more notified cases in the male sex, as observed in this study in which most of the cases in the period, in the microregion, in the state of Espírito Santo, and Brazil, were in the male sex, the epidemic increase is faster in the female sex than male ones in other countries ${ }^{13}$.

One of the ways to study the epidemic feminization is through sex ratio analysis. When the propagation began in 1985, the ratio was 26.5 , but it decreased to 1.4 in 2005 . Recent data reveal that the ratio is still decreasing. In 2009, the ratio was 1.5 cases in men to 1 case in women ${ }^{8}$. In several studies, the ratio decreases between sexes in all of the country areas, showing the well-known epidemic expansion among women ${ }^{8}$.

In this study, similar to the information found in the aforementioned studies, the state of Espírito Santo and Brazil presented decreasing tendencies of sex ratio. Although the microregion has shown increasing tendency of sex ratio, unexpectedly, the determination coefficient was low $\left(\mathrm{R}^{2}=0.3604\right)$, which demonstrates low adjustment to the regression line; the microregion presented the lowest sex ratio average (1.24) when compared with Brazil sex ratio (1.55) and with the state of Espírito Santo (1.3). Besides, the microregion brought the medium occurrence rate of female sex closer to the male one, with only a $2.2 \%$ difference. The state of Espírito Santo and Brazil presented differences of $25.9 \%$ and $37.1 \%$, respectively. Finally, the occurrence rate increase tendency in the female sex in the microregion was the most intense, followed by the state of Espírito Santo and Brazil.

The profile shows that being a woman implicates specificities that present disadvantages, generating a situation of special susceptibility as compared to a man. Women are more vulnerable to HIV infection - biologically, culturally, and socially ${ }^{15}$. However, not all women are vulnerable because there is a differential vulnerability. It is known that the infection affects different women in several ways; it depends on other structural factors ${ }^{5}$.

São Mateus microregion presented occurrence and tendency profiles close to the state's and to the country's, but with peculiarities that prove the accounts. It shows that the AIDS epidemic is characterized by being a dynamic and unstable phenomenon, being translated by a really regional sub-epidemic mosaic, each one of them with its own characteristic.

The decreasing tendency of AIDS occurrence rates in the state of Espírito Santo, apart from the microregion, shows the existence of significant regional differences regarding the epidemic profile. This fact indicates importance for a more careful investigation in the small towns to control AIDS epidemic tendency in those places.

The study reported some of the national-scale transformations with the phenomenon of feminization and interiorization of AIDS-infected people.

Therefore, despite the attained progress in almost 30 years of epidemic, in treatment terms, life quality and prognosis, is important to know that AIDS continues to be an incurable disease and prevention is still the best defense, with specific public policies directed to the epidemic reality in each area.

\section{CONFLICT OF INTEREST}

The authors declare that there is no conflict of interest.

\section{REFERENCES}

1. Brito AM, Castilho EA, Szwarcwald CL. AIDS and HIV infection in Brazil: a multifaceted epidemic. Rev Soc Bras Med Trop 2001; 34: 207-217.

2. Schneider IJC, Ribeiro C, Breda D, Skalinski LM, D'orsi E. Epidemiological profile of the clientele in HIV Testing and Counseling Centers in Santa Catarina State, Brazil, 2005. Cad Saude Publica 2008; 24:1675-1688.

3. Ferreira RCSL, Dias JO, Mello RS, Sakae TM. Epidemiologic profile of AIDS in AMUREL region, South Brazil between 1987 and 2006. Arq Catarin Med 2008; 37:19-24.

4. Sakae TM, Medeiros LS, Peres MAA, Santos R. Profile of mortality for AIDS in Santa Catarina (South Brazil) from 2000 to 2004. Arq Catarin Med 2006; 35:21-27.

5. Parker R, Camargo Jr KR. Poverty and HIV/AIDS: anthropological and sociological aspects. Cad Saude Publica 2000; 16 (sup 1): 89-102. 
6. Ferla AA, Possa LB, Leal M, Valerio AG, Claus SM. Public decentralization and politics for STD/SIDA: Study of the System of Health in the State of Rio of Janeiro.1. ed. Rio de Janeiro: ABIA 2006; vol 1, 42p.

7. Brazil. Health Ministry. Executive Secretary. Department of Informatics of SUS [Internet database] Brasília: Datasus; 1975. [access on 15 feb 2010]. Available from: http://www2.datasus.gov.br/DATASUS/index.php?area=02.

8. Health Ministry. Secretariat of Health Surveillance. National STD And AIDS Program. Epidemiological Bulletin AIDS and STD, year V, $n^{\circ} 1,01^{\mathrm{a}}, 26^{\mathrm{a}}$, epidemiological weeks, jan a jun de 2008.

9. Secretary of State of Health. State Government of Espírito Santo. Special Core of Analysis and Trends in Health Status. Epidemiological Profile of Health in ES: 2006. Vitória: NASTS; 2006. [access on 18 oct. 2010]. Available from: http://www.saude.es.gov.br/default.asp.
10. Cruz CD. Genes program [computer program]: Biometrics. Viçosa: Publisher UFV; 2006.

11. World Health Organization. Joint United Nations Programme on HIV/AIDS. Report on the global AIDS epidemic 2008. Geneva: UNAIDS, 2008

12. Varella RB. Aspects of the AIDS epidemic in a medium-sized municipality of he state of Rio de Janeiro, 2000-2004. Rev Bras Epidemiol 2006; 9: 447-453.

13. Santos NJS, Tayra A, Silva SR, Buchalla CM, Laurenti R. AIDS in the State of São Paulo. Changes in the profile of the epidemic and prospects for epidemiological surveillance. Rev Bras Epidemiol 2002;5: 286-310.

14. Rachid M, Schechter M. Manual of HIV/AIDS. 6 ${ }^{\text {a }}$ ed. Rio de Janeiro (RJ): Revinter; 2001.

15. Bastos FI, Szwarcwald CL. AIDS and pauperization: principal concepts and empirical evidence. Cad Saude Publica 2000; 16 (supl 1): 65-76. 